\title{
Improving metal reflectors by suppressing surface plasmon polaritons: a priori calculation of the internal reflectance of a solar cell
}

\author{
Zachary C Holman, Stefaan De Wolf and Christophe Ballif
}

Imperfect internal reflectance of near-bandgap light reduces the performance of all solar cells, and becomes increasingly detrimental as absorbers become thinner. We consider light incident on the silicon/dielectric/metal structure at the back of rear-passivated crystalline silicon solar cells with surface textures that are large enough for geometric optics. By calculating the absorbance in the metal as a function of the angle of incidence, we discover three results that are important for understanding and improving rear reflectors in many types of solar cells. First, significant parasitic absorption occurs in the metal layer in two cases: $s$ - and p-polarized propagating modes (near-normal angles of incidence) when the dielectric thickness is adjusted to cause destructive interference of the reflected beams, and p-polarized evanescent modes (angles of incidence above the semiconductor/dielectric critical angle) that excite surface plasmon polaritons at the metal surface. Second, the latter loss dominates; a well-designed rear dielectric passivation layer must suppress the penetration of evanescent waves to the metal. Third, when used as an input in a simple analytical model, the average rear internal reflectance calculated by assuming a Lambertian angular distribution of light accurately predicts the total reflectance and absorbance of a solar cell. Light: Science \& Applications (2013) 2, e106; doi:10.1038/lsa.2013.62; published online 25 October 2013

Keywords: light trapping; photovoltaics; plasmon; silicon; solar cell

\section{INTRODUCTION}

The current trend towards higher silicon solar cell efficiencies has brought rear-passivated designs_-including PERC (passivated emitter and rear cell), ${ }^{1}$ interdigitated back contact ${ }^{2}$ and heterojunction ${ }^{3}$ structures - to the forefront of both research and production. While the impetus for rear passivation is a reduction in surface recombination and a corresponding gain in open-circuit voltage $\left(V_{o c}\right)$, the dielectric passivation layer (or transparent conductive oxide layer in silicon heterojunction cells) can be made to simultaneously serve an optical role, thus increasing short-circuit current density $\left(J_{s c}\right)$ too. Several authors have observed that even high-conductivity metals like silver are poor reflectors on textured silicon solar cells, and that a dielectric buffer layer increases the rear internal reflectance of infrared (IR) light. ${ }^{1,4-6}$ In thin-film silicon solar cells that employ nanoscale textures and metallic rear reflectors, transparent conductive oxide (TCO) rear buffer layers suppress localized surface plasmons that are excited in the metal by the rough surfaces. ${ }^{7-10}$ For alkaline-textured monocrystalline wafers with micrometer-sized pyramids, however, a different loss mechanism must be responsible for parasitic absorption in the metal. In optical simulations of planar $\mathrm{Si} / \mathrm{SiO}_{2} / \mathrm{Al}$ structures, Campbell observed diminished internal reflectance both above and below the $\mathrm{Si} / \mathrm{SiO}_{2}$ critical angle for thin $\mathrm{SiO}_{2}$ layers, revealing that evanescent as well as propagating modes may be absorbed in the metal. ${ }^{4,5}$ In a recent investigation of the TCO layers in amorphous silicon/crystalline silicon heterojunction solar cells, we confirmed this finding with both simulations and experiments, and demonstrated that $J_{\text {sc }}$ may be increased by $0.5 \mathrm{~mA} \mathrm{~cm}{ }^{-2}$ with rear TCO layers that minimize the penetration of evanescent waves to the metallic reflector. ${ }^{11}$ In this paper, we expose the physics of parasitic absorption in the metal of rear-passivated solar cells by calculating the electric field intensities in semiconductor/dielectric/metal structures. We then develop a simple formalism for predicting the rear internal reflectance and total IR reflectance of a solar cell given specific dielectric and metal layers, thereby facilitating the optimization of these layers. While only Si/ $\mathrm{SiN}_{x} / \mathrm{Ag}$ is considered here, the results apply equally well to other material systems, including, e.g., III-V solar cells, for which high internal reflectance of near-bandgap photons translates into a $V_{o c}$ as well as a $J_{s c}$ gain because of photon recycling. ${ }^{12}$

\section{MATERIALS AND METHODS}

For a rear-passivated solar cell with a planar rear surface or micrometer-scale features, IR light arriving at the back of the cell sees the locally planar semiconductor/dielectric/metal structure depicted in Figure 1. The coordinate system is arranged such that light polarized perpendicular to the plane of incidence (s-polarized or transverse electric) has its electric field only in the $y$-direction, whereas light

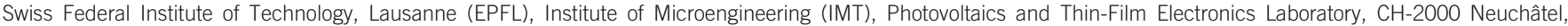
Switzerland

Correspondence: Professor Z Holman, Arizona State University, PO Box 875706, Tempe, AZ 85827-5706, USA

E-mail: zachary.holman@asu.edu

Received 16 January 2013; revised 22 May 2013; accepted 6 July 2013 


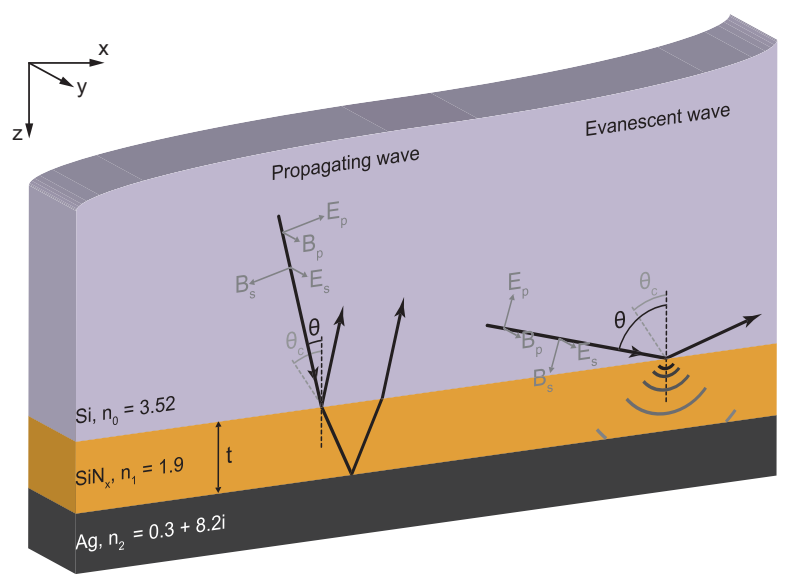

Figure 1 Schematic of the simulated $\mathrm{Si} / \mathrm{SiN}_{x} / \mathrm{Ag}$ structure representing the rear of a solar cell. Rays arriving within (left) and outside (right) the $\mathrm{Si}_{\text {SiN }} \mathrm{S}_{x}$ critical angle are shown, and the fields for both $s$-polarization $\left(E_{s}, B_{s}\right)$ and $p$-polarization $\left(E_{p}, B_{p}\right)$ are indicated to help clarify the coordinate system.

polarized parallel to the plane ( $p$-polarized or transverse magnetic) has components in both the $x$ - and $z$-directions. The electric field intensities throughout the structure are defined as

$$
\begin{aligned}
& F_{x}(z)=\frac{\left|E_{x}(z)\right|^{2}}{\left|E_{0 p}\right|^{2}}, \\
& F_{y}(z)=\frac{\left|E_{y}(z)\right|^{2}}{\left|E_{0 s}\right|^{2}}, \\
& F_{z}(z)=\frac{\left|E_{z}(z)\right|^{2}}{\left|E_{0 p}\right|^{2}}
\end{aligned}
$$

where $E_{j}(z)$ is the electric field in the $j$ th direction as a function of position $z$, and $E_{0 s}$ and $E_{0 p}$ are the incident $s$ - and $p$-polarized fields. We calculated the field intensities with the explicit formulas given by Ohta and Ishida-which were derived with the transfer matrix method-as well as the Fresnel transmission and reflection coefficients. ${ }^{13}$ The thickness $t$ of the dielectric layer was varied-this is the parameter that is easiest to change in a solar cell-and the influence of the angle of incidence $\theta$ was studied. All calculations were performed in Mathematica.

For silicon wafer-based solar cells, photons with wavelengths of 900-1150 $\mathrm{nm}$ are above the bandgap yet sufficiently weakly absorbed that they reach the rear side of the cell at least once, making them susceptible to parasitic absorption in the metal reflector. A wavelength of $1200 \mathrm{~nm}$ was chosen for all calculations because it is representative of the wavelength range of interest, but is just below the bandgap, and thus is not absorbed in the silicon itself. The semiconductor, dielectric and metal refractive indices were assumed to be $n_{0}=3.52, n_{1}=1.9$ and $n_{2}=0.3+8.2 i$, respectively; these correspond to the values for $\mathrm{Si}, \mathrm{SiN}_{x}$ and Ag at $1200 \mathrm{~nm} .{ }^{14,15}$ Although many PERC-like cells have aluminum reflectors, silver was chosen to demonstrate how lossy even 'good' metals can be. Changing the wavelength or refractive indices alters the values of the quantities calculated in this paper, but not the underlying physics.
From the angle-dependent reflection loss per internal reflection at the rear reflector, we calculated the average rear internal reflectance $r_{r}$ by integrating over a Lambertian angular distribution function. $\mathrm{SiN}_{x} /$ $\mathrm{Si} / \mathrm{SiN}_{x} / \mathrm{Ag}$ solar-cell-like structures were fabricated with select rear $\mathrm{SiN}_{x}$ thicknesses, and their total reflectance spectra measured. We then simulated the same spectra using as input the calculated $r_{r}$ values for the measured $\mathrm{SiN}_{x}$ thicknesses, and compared the measured and calculated spectra to evaluate our model for $r_{r}$. Though $s$ - and $p$-polarized light are treated separately throughout much of the paper, we used unpolarized light when simulations were compared to experiments.

To fabricate the $\mathrm{SiN}_{x} / \mathrm{Si} / \mathrm{SiN}_{x} / \mathrm{Ag}$ structures, silicon wafers (float zone, (100), $n$-type, $3 \Omega \mathrm{cm}, 280 \mu \mathrm{m}$ ) were textured in an alkaline bath to create random pyramids with feature sizes of approximately $5 \mu \mathrm{m}$ on both surfaces. Subsequently, $\operatorname{SiN}_{x}$ layers were deposited on the front and rear of each wafer by plasma-enhanced chemical vapor deposition, and a silver layer was deposited on the rear by directcurrent sputtering. The front $\mathrm{SiN}_{x}$ layer was $70 \mathrm{~nm}$ thick, the rear $\mathrm{SiN}_{x}$ thickness varied, and the Ag layer was approximately $300 \mathrm{~nm}$ thick. A flat glass sample witnessed each deposition; the thicknesses of the layers were measured with profilometry on these witnesses and the results were divided by 1.7 to obtain the layer thicknesses on the textured silicon surfaces. This factor reflects the fact that the same flux of atoms must coat a larger surface on the textured wafers, and it was verified by comparing the interference extrema of codeposited layers on polished and textured wafers. Spectroscopic ellipsometry of $\mathrm{SiN}_{x}$ layers on the witness substrates revealed that $n_{1}=1.9$ at $1200 \mathrm{~nm}$, the value used in calculations. The total reflectance spectra of these structures were measured with a dual-beam spectrophotometer equipped with an integrating sphere using unpolarized light.

The reflectance spectra of the same structures were simulated with a Lambertian analytical model proposed by Boccard et al. that extends the formalism introduced by Deckman et al. ${ }^{16,17}$ In this model, the wafer is treated as a planar semiconductor slab with front internal reflectance $r_{f}$ and rear internal reflectance $r_{r}$. The model also allows for a different front internal reflectance $r_{f, f i r s t}$ the first time IR light returns to the front surface after one round trip through the cell. This parameter, the only one in the model that was fit, takes into account the fact that the angular distribution function may be narrower than Lambertian during the first few passes, causing more light to escape from the front of the cell. In the model, light travels a distance of twice the wafer thickness between reflection events, corresponding to the average path for a Lambertian distribution. As this beam of 'average rays' bounces back and forth in the wafer, losing intensity at each surface, the escaping rays are summed and added to the primary front reflectance to yield the total reflectance.

\section{RESULTS AND DISCUSSION}

Maps of the calculated field intensities at the rear of a silicon solar cell are plotted in Figure 2 as a function of $\theta$ and $z$ for $\operatorname{SiN}_{x}$ thicknesses of 20, 200 and $1000 \mathrm{~nm} . F_{x}(z)$ and $F_{y}(z)$ are continuous at the interfaces, as required by the boundary conditions, but $F_{z}(z)$ is discontinuous across changes in dielectric function. Standing waves are present in the silicon, as well as in the thickest $\operatorname{SiN}_{x}$ layer for $\theta<\theta_{c}$, where $\theta_{c}$ is the $\mathrm{Si}$ / $\mathrm{SiN}_{\mathrm{x}}$ critical angle denoted by the vertical dashed lines. For $\theta>\theta_{c}$, the field becomes evanescent and decays exponentially into the $\operatorname{SiN}_{x}$. The characteristic penetration depth of the evanescent wave is ${ }^{18}$

$$
d_{p}(\theta)=\frac{\lambda}{2 \pi n_{0} \sqrt{\sin ^{2} \theta-\left(n_{1} / n_{0}\right)^{2}}}
$$



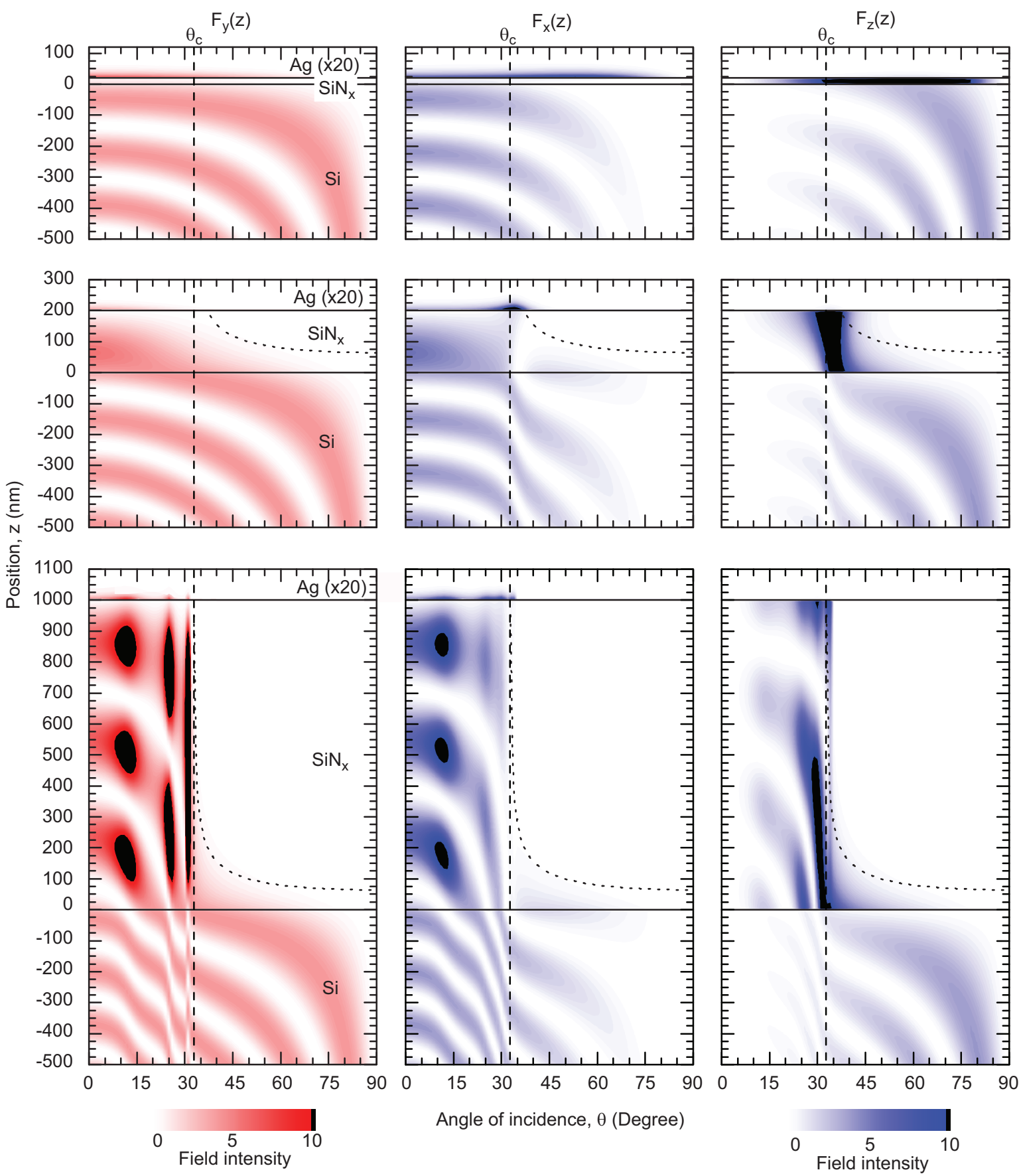

Figure $2 y$-, $x$ - and $z$-components of the field intensity of $1200 \mathrm{~nm}$ light in Si/SiN $/$ /Ag structures with $\mathrm{SiN}_{x}$ layers $20 \mathrm{~nm}$ (top row), $200 \mathrm{~nm}$ (middle row) and $1000 \mathrm{~nm}$ (bottom row) thick. Note that $F_{y}(z)$ is in the left column because the $p$-polarized field intensity is the sum of $F_{x}(z)$ and $F_{z}(z)$, and it is therefore visually preferable to have them adjacent. The field intensities in the silver layer are multiplied by 20 for clarity. The vertical dashed lines denote the $\mathrm{Si}_{\mathrm{SiN}} \mathrm{N}_{x} \mathrm{Critical}$ angle and the dotted lines indicate the characteristic decay length of the evanescent wave given by Equation (2).

with $\lambda$ the wavelength in air. Equation (2) is plotted as a dotted line in Figure 2. The $z$-component of the field is very strong in thin $\operatorname{SiN}_{x}$ layers (more than $30 \times$ enhancement; top-right map) for a wide range of angles, and is concentrated near the critical angle for thicker layers. Consequently, if the dielectric layer is thin and has non-zero extinction, it will strongly absorb $p$-polarized light at high angles of incidence. We observed this in the rear TCO in silicon heterojunction solar cells; ${ }^{11}$ it is also the principle behind metal overlayer-attenuated total reflection, an IR spectroscopic technique to enhance the absorption of ultrathin layers under investigation. ${ }^{19,20}$ Most interesting for this study, $F_{z}(z)$ is near-zero at the metal surface; instead, $F_{x}(z)$, and to a lesser extent $F_{y}(z)$, is resonant with the metal.

The field intensities at the silver surface are summarized in Figure 3 for $s$-polarized $\left(F_{y}(z)\right)$ and $p$-polarized $\left(F_{x}(z)+F_{z}(z)\right)$ light for several $\mathrm{SiN}_{x}$ thicknesses. As is also apparent from the top row of Figure 2, the field intensity at the metal surface is relatively strong for $p$-polarized light incident on thin dielectric layers with a wide range of angles of incidence above the critical angle. This is the signature of surface 


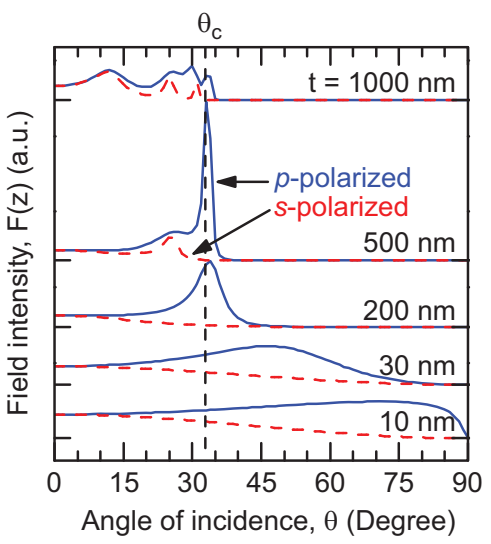

Figure 3 Field intensities of $1200 \mathrm{~nm} s$ - and p-polarized light at the silver surface for varying $\mathrm{SiN}_{x}$ thicknesses. The curves are offset vertically for clarity. The vertical dashed line denotes the $\mathrm{Si}_{/} \mathrm{SiN}_{x}$ critical angle.

plasmon polaritons: spatially extended oscillations of charge at the metal surface. Although plasmons cannot be excited by propagating modes on planar surfaces, Otto demonstrated that they can be excited across a low-refractive-index layer by evanescent modes of $p$-polarized light. ${ }^{21}$ The rear structure of rear-passivated solar cells may thus be recognized as unintentionally having the so-called Otto configuration. Figure 3 shows that the plasmon resonance shifts to smaller angles with increasing $\mathrm{SiN}_{x}$ thickness and settles just above the critical angle. This is because plasmons are excited only for $\theta_{c}<\theta<\theta_{d p}$ where $\theta_{d p}$ is defined by $d_{p}\left(\theta_{d p}\right)=t$; for larger angles, the evanescent wave decays too quickly to see the metal layer (see Figure 2, bottom row). There are also strong fields just below $\theta_{c}$ for $p$-polarized light and some $\operatorname{SiN}_{\mathrm{x}}$ thicknesses (e.g., $200 \mathrm{~nm}$ ). This light reaches the silver at grazing angles near the pseudo-Brewster angle, for which $p$ - but not $s$-polarized light is coupled to the metal. For $\theta<20^{\circ}$, the field at the metal surface is similar for $s$ - and $p$-polarized light, and may be large at specific angles when the dielectric layer is several hundreds of nanometers thick. By studying the standing waves in Figure 2, it becomes clear that these fields are caused by the destructive interference of reflected propagating modes; the dielectric layer is behaving as an antireflection coating, increasing the intensity of the standing waves within the dielectric and coupling light to the silver reflector.

The absorbance $\mathrm{d} A$ in a layer of refractive index $n$ and infinitesimal thickness $\mathrm{d} z$ is proportional to the product of the field intensity and the layer's absorption coefficient $\alpha:^{13}$

$$
\mathrm{d} A=\frac{n}{n_{0} \cos \theta} \alpha F(z) \mathrm{d} z
$$

The parasitic absorbance in the silver reflector may thus be calculated by integrating Equation (3) over the layer thickness (a few tens of nanometers is sufficient for metals). Alternatively, the absorbance may be calculated using the Fresnel transmission and reflection coefficients. ${ }^{13}$ This approach is faster and gives the same result, but does not provide the physical insight that comes with plotting the field intensities.

The silver absorbance for a single rear internal reflection is displayed as a map in Figure 4. As anticipated by Equation (3), the absorbance tracks the field intensities displayed in Figure 3: p-polarized light is strongly absorbed for $\theta>\theta_{c}$ (due to plasmon excitation) and the absorbance peak narrows and shifts towards the critical angle with increasing $\mathrm{SiN}_{x}$ thickness, whereas the absorbance of $s$ - and $p$-polarized light

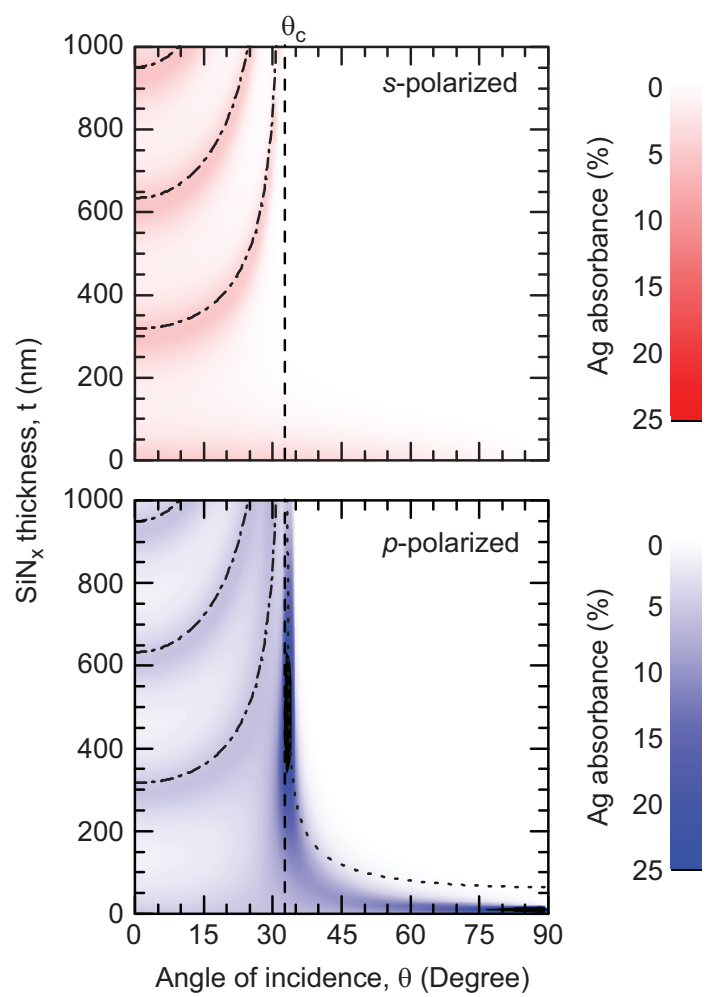

Figure 4 Absorbance of $1200 \mathrm{~nm}$ s- and p-polarized light in the silver layer per internal reflection at the rear surface for $\mathrm{SiN}_{x}$ layers of varying thickness. The vertical dashed lines denote the $\mathrm{Si} / \mathrm{SiN}_{x}$ critical angle and the dotted line indicates the characteristic decay length of the evanescent wave given by Equation (2). The dash-dotted lines indicate the solutions to Equation (5), which correspond to destructive interference conditions.

is similar for $\theta<\theta_{c}$ and is attributable to interference. The role of interference may be confirmed by considering that the primary and secondary reflected beams are out of phase when

$$
\left(m+\frac{\pi+\varphi_{1}+\varphi_{2}}{2 \pi}\right) \lambda=2 n_{1} t \cos \left[\sin ^{-1}\left(\frac{n_{0}}{n_{1}} \sin \theta\right)\right]
$$

where $\varphi_{1}$ and $\varphi_{2}$ are the phase shifts in radians at the first and second interfaces, respectively, $m$ is an integer and the right-hand side expresses the optical path difference of the two beams. Given that $\varphi_{1}=0$ and $\varphi_{2}=\pi$ for our $\mathrm{Si} / \mathrm{SiN}_{x} / \mathrm{Ag}$ structure, destructive interference-and consequently increased silver absorbance-occurs for $\mathrm{SiN}_{\mathrm{x}}$ thicknesses of

$$
t(\theta)=\frac{(m+1) \lambda}{2 n_{1}}\left\{\cos \left[\sin ^{-1}\left(\frac{n_{0}}{n_{1}} \sin \theta\right)\right]\right\}^{-1}
$$

The solutions to Equation (5) are plotted in Figure 4 as dash-dotted lines, and they align with the calculated absorbance maxima for propagating modes. As in Figure 2, the evanescent wave penetration depth given by Equation (2) is plotted as a dotted line for $p$-polarized light. The absorbance in the silver reflector is approximately bounded by $d_{p}(\theta)$ because, as stated previously, the evanescent wave does not penetrate to the metal for $\theta$ that satisfy $d_{p}(\theta)<t$. Note that Figure 4 could in principle be reproduced experimentally by illuminating silicon hemispheres coated on their flat sides with $\mathrm{SiN}_{x} / \mathrm{Ag}$ layer stacks with an IR laser and varying the angle of incidence (surface-plasmon spectroscopy). 


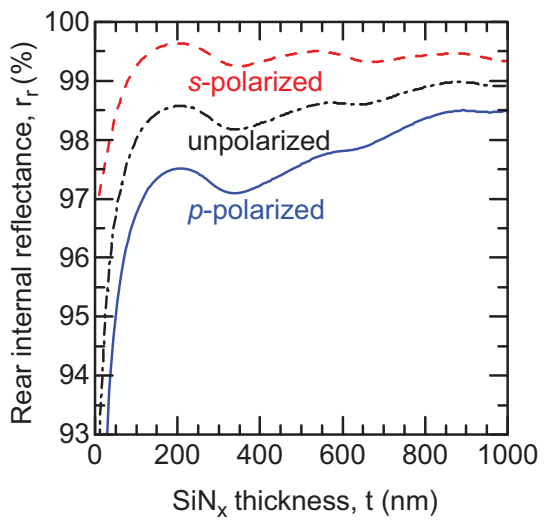

Figure 5 Average rear internal reflectance of $1200 \mathrm{~nm}$ light assuming a Lambertian angular distribution function.

What does this mean for solar cells? A textured solar cell may be modeled as a planar device with surfaces that scatter light into the same distribution of angles that the texture does on average. In the simplest approximation, the surfaces scatter light randomly, generating a Lambertian angular distribution function $(A D F)$ :

$$
A D F(\theta)=\frac{1}{2} \cos \theta
$$

In real silicon wafer-based solar cells with random pyramids, light becomes near-Lambertian only after a few scattering events; ${ }^{22}$ nevertheless, we will see that this oft-employed assumption suffices for our purposes. Integrating the absorbance in Figure 4, weighted by the angular distribution function, over $\theta$ yields $1-r_{r}$, where, as before, $r_{r}$ is the average rear internal reflectance (per interaction with the rear surface). $r_{r}$ is plotted in Figure 5 as a function of $\mathrm{SiN}_{x}$ thickness. The most dramatic feature is the severe drop in internal reflectance for $t<100 \mathrm{~nm}$ because of plasmonic absorption. Although Figure 4 indicates strong absorption of $p$-polarized light near the critical angle for layers several hundreds of nanometers thick, the effect on the average internal reflectance is small because the absorption occurs only for a narrow range of angles. The best dielectric layer is thick enough to minimize the penetration of $p$-polarized evanescent fields to the metal reflector (approximately $200 \mathrm{~nm}$ for this materials system); thicker layers result in little gain and are technologically impractical. Figure 5 also shows that when rear $\mathrm{SiN}_{x}$ passivation layers are designed for optical performance, internal reflectances of $98.5 \%$ are achievable (noting that sunlight is unpolarized), minimizing IR parasitic absorption and boosting $J_{s c}$.

Common solar cell analysis programs such as $\mathrm{PC}^{2} \mathrm{D}^{23}$ calculate the total reflectance $R$ and external quantum efficiency $(E Q E)$ of a solar cell using $r_{r}$ as an input. In many cases, users have no a priori knowledge of internal reflectance, and they thus estimate it using a guessand-check approach until a calculated spectrum matches a measured spectrum. The internal reflectance shown in Figure 5, which was derived using only refractive indices, may be used directly in optical models.

To test the accuracy of this approach, we simulated the total reflectance spectra of $\mathrm{SiN}_{x} / \mathrm{Si} / \mathrm{SiN}_{x} / \mathrm{Ag}$ solar cell-like structures and compared the results to measurements. These structures represent solar cells in which the emitter and back-surface field (possible sources of free-carrier absorption) have been omitted, so that parasitic absorption at $1200 \mathrm{~nm}$ occurs only in the silver reflector (note that, with a doping density of $1.5 \times 10^{15} \mathrm{~cm}^{-3}$, the wafer itself has a free-carrier

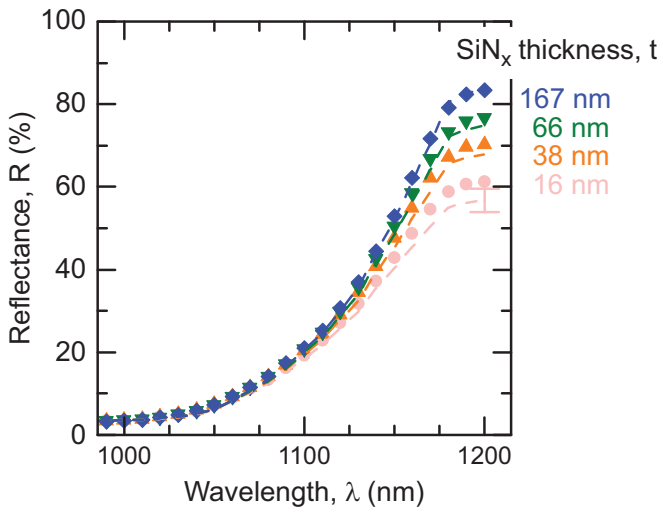

Figure 6 Measured (symbols) and simulated (dashed lines) total reflectance of an alkaline-textured silicon wafer with a $70-\mathrm{nm}$-thick front $\mathrm{SiN}_{x}$ layer, a variablethickness rear $\mathrm{SiN}_{x}$ layer and a silver rear reflector.

absorption coefficient of $2 \times 10^{-3} \mathrm{~cm}^{-1}$ at $1200 \mathrm{~nm}$ and absorbs only $0.01 \%$ of light per pass $^{24}$ ). The measured spectra are shown with symbols in Figure 6. The sub-bandgap reflectance (i.e., $R$ at $1200 \mathrm{~nm}$ ) increases with $\operatorname{SiN}_{x}$ thickness for thicknesses up to $167 \mathrm{~nm}$, indicating diminishing parasitic absorption in the silver layer. This is expected from the above simulations identifying plasmonic excitation. The reflectance spectra of structures with thicker $\operatorname{SiN}_{x}$ layers nearly overlap that of the $167 \mathrm{~nm}$ sample (not shown). The simulated spectra are plotted as dashed lines in Figure 6. The spectra were generated with the analytical Lambertian model developed by Boccard et al. ${ }^{16}$ using the $r_{r}$ values from Figure 5 that correspond to the measured rear $\mathrm{SiN}_{x}$ thicknesses (93.6, 96.1, 97.3 and 98.5\% for layers $16,38,66$, and $167 \mathrm{~nm}$ thick, respectively). While these $r_{r}$ values are, strictly speaking, specific to $1200 \mathrm{~nm}$ light, they change by less than $0.5 \%$ as the wavelength decreases to $1000 \mathrm{~nm}$. This relative insensitivity to small variations in wavelength allows us to simulate the total reflectance spectra without recalculating $r_{r}$. PC1D or many other simulation tools could have been used instead of Boccard's model; we chose this model because it has the fewest fitting parameters and thus would not allow us to compensate with other parameters if the calculated $r_{r}$ values were incorrect. In accordance with a Lambertianscattering surface, $r_{f}$ was set to $1-1 / n_{0}{ }^{2}=91.9 \%$. We found $r_{f, f i r s t}=90 \%$ to give the best fit to the measured data, though $r_{f, f i r s t}=r_{f}=91.9 \%$ did not change the reflectance dramatically.

The calculated curves fit the measured data remarkably well given the Lambertian assumption and the simplicity of the analytical model, faithfully reproducing the shape of the spectra. For the thinner $\operatorname{SiN}_{x}$ layers, the measured and simulated curves slightly deviate above $1100 \mathrm{~nm}$. This may be due to small errors in the thicknesses measured by profilometry, which would result in large errors in rear internal reflectance for thin $\mathrm{SiN}_{x}$ layers. The error bar provided for the $16 \mathrm{~nm}$ sample indicates the change in calculated reflectance at $1200 \mathrm{~nm}$ if $t$ is varied by $\pm 5 \mathrm{~nm}$. Because $r_{r}$ may be used in a simple model to accurately predict $R$, it may also be used to predict EQE (in our model, assuming perfect collection, by summing the rays absorbed in the wafer), and thus $J_{s c}$. This provides a clear path to $J_{s c}$ improvement in rear-passivated solar cells, as their IR response may be calculated and subsequently optimized given only the semiconductor, dielectric and metal refractive indices, and the dielectric thickness.

\section{CONCLUSIONS}

Solar cells with dielectric/metal rear reflectors suffer from imperfect internal reflection, which can be understood by calculating the electric 
fields in these structures. Most striking, when the dielectric layer is thin, the evanescent field of $p$-polarized light excites surface plasmon polaritons for a wide range of angles of incidence above the critical angle. The resulting absorption in the metal is a major loss mechanism for near-bandgap light, and any reflector design should seek foremost to mitigate this absorption. The present investigation was limited to a single, representative materials system, for which we demonstrated that absorption in the silver reflector is suppressed by increasing the $\mathrm{SiN}_{x}$ thickness. Nevertheless, other materials systems (including direct-bandgap solar cells) and other approaches (such as reducing the dielectric refractive index) may be effectively explored and quantified within the framework introduced here.

\section{ACKNOWLEDGMENTS}

We thank Miha Filipič and Mathieu Boccard for helpful discussions. This work was supported by the European Union Seventh Framework Programme (FP7/ 2007-2013), Collaborative Project '20pl $\mu$ s' with the full title: 'Further development of very thin wafer based c-Si photovoltaics' under grant agreement no. 256695, by Axpo Naturstrom Fonds, Switzerland, and by the Swiss Commission for Technology and Innovation.

1 Blakers AW, Wang A, Milne AM, Zhao JH, Green MA. 22.8\% efficient silicon solar cell. Appl Phys Lett 1989; 55: 1363-1365.

2 Lammert MD, Schwartz RJ. The interdigitated back contact solar cell: a silicon solar cell for use in concentrated sunlight. IEEE Trans Electron Dev 1977; 24: 337-342.

3 Tanaka M, Taguchi M, Matsuyama T, Sawada T, Tsuda S et al. Development of new aSi/c-Si heterojunction solar cells: ACJ-HIT (artificially constructed junctionheterojunction with intrinsic thin-layer). Jpn J Appl Phys 1992; 31: 3518-3522.

4 Campbell P.Light trapping and reflection control in a crystalline silicon solar cell. PhD thesis, University of New South Wales, Kensington, NSW, Australia, 1989.

5 Green MA. Silicon Solar Cells: Advanced Principles \& Practice. Sydney: University of New South Wales, 1995.

6 Kray D, Hermle M, Glunz SW. Theory and experiments on the back side reflectance of silicon wafer solar cells. Prog Photovoltaics Res App/ 2008; 16: 1-15.

7 Ross R, Mohr R, Fournier J, Yang J. Status of fluorinated amorphous silicongermanium alloys and multijunction devices. In: Proceedings of the 19th IEEE
PVSC; 4-8 May 1987; New Orleans, LA, USA. IEEE: New York, USA, 1987 pp327-330.

8 Kothandaraman C, Tonon T, Huang C, Delahoy AE. Improvement of a-Si:H p-i-n devices using zinc oxide based back-reflectors. MRS Proc 1991; 219: 475-480.

9 Haug FJ, Soderstrom T, Cubero O, Terrazzoni-Daudrix V, Ballif C. Plasmonic absorption in textured silver back reflectors of thin film solar cells. J Appl Phys 2008; 104: 064509.

10 Muller J, Rech B, Springer J, Vanecek M. TCO and light trapping in silicon thin film solar cells. Sol Energy 2004; 77: 917-930.

11 Holman ZC, Filipic M, Descoeudres A, de Wolf S, Smole F et al. Infrared light management in high-efficiency silicon heterojunction and rear-passivated solar cells. J Appl Phys 2013; 113: 013107.

12 Miller OD, Yablonovitch E, Kurtz SR. Strong internal and external luminescence as solar cells approach the Shockley-Queisser limit. IEEE J Photovoltaics 2012; 2: 303311.

13 Ohta K, Ishida H. Matrix formalism for calculation of electric field intensity of light in stratified multilayered films. Appl Opt 1990; 29: 1952-1959.

14 Jellison GE Jr. Optical functions of silicon determined by two-channel polarization modulation ellipsometry. Opt Mater 1992; 1: 41-47.

15 Palik ED (ed). Handbook of Optical Constants of Solids. San Diego, CA: Academic Press, 1997.

16 Boccard M, Battaglia C, Haug FJ, Despeisse M, Ballif C. Light trapping in solar cells: analytical modeling. App/ Phys Lett 2012; 101: 151105.

17 Deckman HW, Wronski CR, Witzke H, Yablonovitch E. Optically enhanced amorphous silicon solar cells. App/ Phys Lett 1983; 42: 968-970.

18 Harrick NJ, Dupre FK. Effective thickness of bulk materials and of thin films for internal reflection spectroscopy. Appl Opt 1966; 5: 1739-1743.

19 Ishino Y, Ishida H. Grazing angle metal-overlayer infrared ATR spectroscopy. App/ Spectrosc 1988; 42: 1296-1302.

20 Hatta A, Ohshima T, Suetaka W. Observation of the enhanced infrared absorption of $p$ nitrobenzoate on Ag island films with an ATR technique. App/ Phys A 1982; 29: 7175.

21 Otto A. Excitation of nonradiative surface plasma waves in silver by the method of frustrated total reflection. Z Phys 1968; 216: 398-410.

22 Campbell P, Green MA. Light trapping properties of pyramidally textured surfaces. $J$ Appl Phys 1987; 62: 243-249.

23 Basore PA. Numerical modeling of textured silicon solar cells using PC-1D. IEEE Trans Electron Dev 1990; 37: 337-343.

24 Schroder DK, Thomas RN, Swartz JC. Free carrier absorption in silicon. IEEE Trans Electron Dev 1978; 25: 254-261.

(c) (i) (5) This work is licensed under a Creative Commons AttributionBY NC SA NonCommercial-ShareAlike 3.0 Unported license. To view a copy of this license, visit http://creativecommons.org/licenses/by-nc-sa/3.0 